Wang R., Jia D., Zhang W. and Tong Y. (2020). "3D reverse geometrical modeling and Building Information Modeling of historic buildings" In: Proc. 37th CIB W78 Information Technology for Construction Conference (CIB W78), São Paulo, Brazil, pp. 266-278. DOI: http://dx.doi.org/10.46421/27066568.37.2020.paper019

\title{
3D REVERSE GEOMETRICAL MODELING AND BUILDING INFORMATION MODELING OF HISTORIC BUILDINGS
}

\author{
Ruilin Wang ${ }^{1}$, Dongfeng Jia ${ }^{2}$, Weiping Zhang ${ }^{3}$, and Yu Tong ${ }^{4}$
}

\begin{abstract}
Historic buildings combining functional, historic, scientific, art and social values, deserve scientific conservation. Thus, it is of significance to establish the information archiving. This paper mainly focuses on 3D reverse modeling and building information modeling of historic buildings, laying a foundation for digital archiving and life-cycle maintenance management.

According to the conservation requirements and data characteristics for different parts of historic buildings, three-dimensional laser scanning, UAV aerial photography and close-range photography are selected to acquire point cloud model. And then, the 3D geometric model of a historical building can be created by 3DS Max and Geomagic, and integrated in 3DS Max.

In order to solve the problem of poor generality and large amount of data of geometric model created by reverse modeling technology, an efficient way to transform geometric model to BIM is proposed. Components are automatically identified and classified according to their characteristics with Maxscript language, and the geometric information of components is imported into text files according to the syntax of component's creation in Revit API. Then, a program called Revit API was developed to read the text files and to fulfill data com-pression through parameter analysis of components in historical buildings, and the 3D geometrical model of the historical building was automatically trans-formed into a building information model, which can integrate many types of information such as history, structure and materials.

Finally, taking Shanghai Jade Buddha Temple as an example to verify the validity of the above procedure. Its BIM model is successfully generated and has been developed into a $4 \mathrm{D}$ model by integrating the past repair and rehabilitation information.
\end{abstract}

Keywords: Historic Building, Reverse Geometric Modeling, Building Information Model, Data Compression, Clustering Algorithm.

\section{INTRODUCTION}

The lack of original design drawings and technique documents is a common and tough issue during conservation, rehabilitation and utilization of historic buildings. Research work has been carried out to establish the information archiving of historical

1 Mr., Key Laboratory of Performance Evolution and Control for Engineering Structures of Ministry of Education, Tongji University, Shanghai, China, ruilin_wang@foxmail.com

2 Dr., Key Laboratory of Performance Evolution and Control for Engineering Structures of Ministry of Education, Tongji University, Shanghai, China, sosokd7@163.com

3 Prof., Key Laboratory of Performance Evolution and Control for Engineering Structures of Ministry of Education, Tongji University, Shanghai, China, weiping zh@tongji.edu.cn

4 Mr., Key Laboratory of Performance Evolution and Control for Engineering Structures of Ministry of Education, Tongji University, Shanghai, China, tytongyu@163.com 
buildings around the world. Conventionally, manual field survey is employed to acquire historical building information(Eslami et al., 2012). Substantial amounts of labor and time are apparently required for historical buildings with irregular shape, complicated texture and structure.

In order to solve this problem, reverse modeling technologies such as 3D laser scanning, Structure from Motion (SfM) also known as terrestrial laser scanning (TLS) and photogrammetry, are used to acquire the information. Agarwal used GPUaccelerated and distributed SfM to reconstruct city-scale point cloud with more than a hundred thousand images of Roma in less than a day and successfully collected geometry information, demonstrates the efficiency of this method(Agarwal et al., 2011). Klein and Bhatla both employed the above image-based method to get point cloud, and further generated $\mathrm{CAD}$ model by picking reference points and joining them using lines manually, their result indicated that extracted dimensions were with error about 3\%7\%(Bhatla et al., 2012; Klein et al., 2012). Compared with image-based point cloud, laser scanned point cloud has higher accuracy. Golparvar-Fard proved the advantage of 3D laser scanning by comparing eight sets of point cloud models generated by above two methods, the result showed that the accuracy of the image-based point cloud is less than that generated by the laser scanner in both laboratory and actual field experiments(Golparvar-Fard et al., 2011). This method also has limitations, for example, laser scanners are too costly and heavy, causing inconvenience to move around for complete information. Therefore, there were some studies trying to combine these two methods for overcome their own shortcomings. Yastikli proposed the whole documentation process to combine digital photogrammetry and TLS, including data acquisition, data processing and final product, and the resulting textured surface model of the Dolmabahce Palace dome were rich in detail information(Yastikli, 2007). Lerma chose the Upper Palaeolithic Cave of Parpallo as the research object, and with the combined use of close-range photogrammetry and TLS, obtained the textured surface model and successfully exported the elevation plan of the cave(Lerma et al., 2010). Xu and Torres-Martínez also performed the similar modeling workflow(Torres-Martínez et al., 2015; Xu et al., 2014). These studies verify reverse modeling based on multi-source data is a promising idea, lay a solid foundation for the efficient information archiving of historical buildings. However, the generated geometric model only contains vertex with a huge size of data, and more valuable information such as structural monitoring data, history and art, is not included in the model. Most important of all, the model format cannot meet the requirement of information sharing, causing limitations in applications.

Building information modeling (BIM) is suitable for this situation, because BIM model can carry heterogeneous and multi-layered information beyond geometric characteristics, and support a common data structure called Industry Foundation Classes (IFC) which guarantees the exchange of relevant data between different software platforms, improving the generality of the model and ensuring quality and efficiency of the historical building's conservation(Saygi et al., 2013). Biagini presented a BIM-based implementation for the documentation of architectural heritage, they captured data using TLS and photogrammetry, and chose Revit and Autocad for creating solid model and integrated them in Revit, because BIM software require the model consists of solid components, instead of vertex in original geometric model(Biagini et al., 2016). Need to mention that there are many other auxiliary tools can help to create non-standard architectural objects based on point cloud or surface model, such as Rhino, 3Ds MAX, SketchUp(López et al., 2018). After modeling in BIM software, there are many useful applications of BIM model: Crespi collected structural information of an historical 
masonry building using non-destructive tests (NDT) and minor destructive tests, and import the BIM model into Midas FEA for structural analysis(Crespi et al., 2015); Wang designed a BIM-based model to support fire safety management of buildings, which was capable of evacuation assessment, escape route planning, safety education, and equipment maintenance(Wang et al., 2015); Theiler extended the IFC schema to describe monitoring-related information for BIM-based modeling of structural health monitor (SHM) systems, and did experiment with laboratory test structure, verified the validation of the proposed IFC model on SHM(Theiler and Smarsly, 2018). These works demonstrate that BIM model can provide comprehensive data shared among different platforms for further applications and surely can make conservation more efficient.

Based on these previous researches, this study proposes a new workflow on multisource data based reverse modeling and an automatic transformation program to bridge the gap between geometric model and information model. And the informatization of Jade Buddha Temple is taken as an example to show the feasibility of the procedure and the wide application of its information model.

\section{REVERSE RECONSTRUCTION OF GEOMETRIC MODEL BASED ON MULTI-SOURCE DATA}

\subsection{Multi-source Data Collection}

Considering the complex shape of historical buildings, data should be collected with multiple methods, ensuring data integrity and accuracy. In this study, a 3D laser scanner was used to complete the overall scanning of the main part of the building; The handheld scanner and camera were used to focus the sampling of architectural details (such as carvings, textures, etc.) to improve the accuracy of model; The UAV collected data of hidden part such as the historical buildings' roof to make up for missing data.

\subsection{Data Processing}

\subsubsection{Structure from Motion and Multi View Stereo}

SfM and MVS are classical computer vision technologies. The geometric relationship between image points and object points can be solved based on corresponding image points, so as to obtain a dense point cloud of the measured object, as shown in Figures 1.

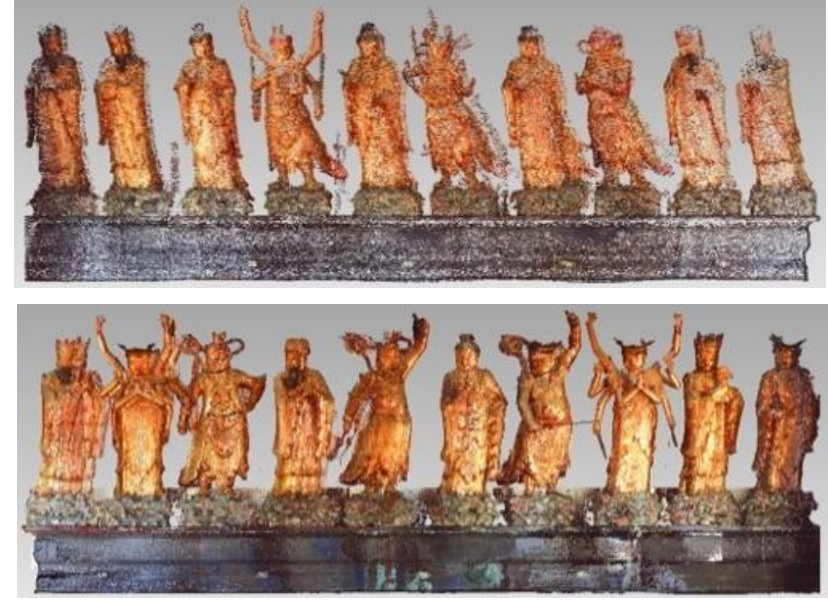

Figure 1: Twenty Heaven Statue in the Mahavira Hall. 


\subsubsection{Point Cloud Filtering and Reduction}

Based on the original point cloud, data filtering algorithm can be used to effectively remove noise points. In addition, it is also necessary to comprehensively use the curvature sampling method and the preserved boundary method to reduce the huge point cloud data (Lee et al., 2001).

\subsubsection{Multi-source Data Fusion}

Through the data processing introduced above, the geometric information in the form of point cloud can be obtained. However, for the geometric information obtained by different methods, there are still problems such as data separation, excessive data volume, and lack of topological relationships, which need to be solved by the following ways.

\subsubsection{Point Cloud Fusion}

Referring to the relevant theories of point cloud data registration and digital image matching, the point cloud collected by the 3D laser scanner and the digital image captured by the camera also have a lot of overlapping parts. Through searching for corresponding points, the rigid body transformation of the data in different coordinate systems can be carried out, and the 3D laser scanning point cloud data and image point cloud data can be merged together. Figure 2 shows point cloud of the Mahavira Hall.

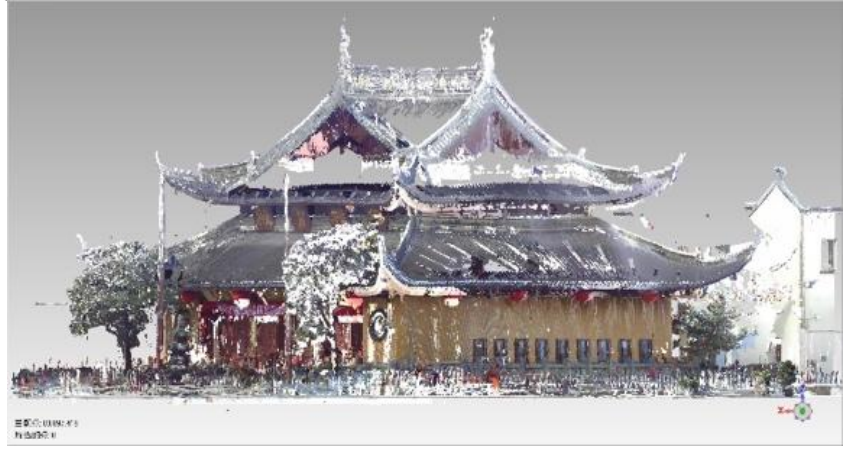

Figure 2: Point cloud of the Mahavira Hall.

\subsubsection{Point Cloud Segmentation}

Although the noise and the density of point cloud data have been effectively reduced after data filtering and data reduction, the fused point cloud data still inevitably has a problem of excessive data size. Reasonable segmentation of the point cloud data is needed to facilitate subsequent applications. In this study, segmentation is performed by edge-based and region-based segmentation algorithms and manual operation in a point cloud post-processing software called Geomagic. In this way, the point cloud data of the Mahavira Hall can be segmented into two parts, the roof structure and the substructure, as shown in Figure 3.

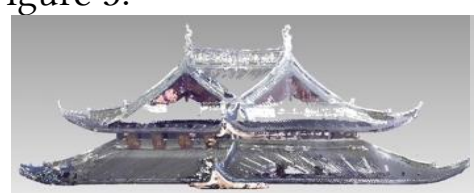

(a)

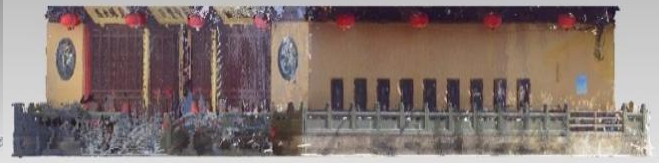

(b)

Figure 3: (a) Point cloud of roof. (b)Point cloud of substructure.

\subsubsection{Polygon Mesh.}

Although high-density point cloud data can express the three-dimensional geometric information of historical buildings, it is still a large number of dense discrete points, 
which don't belong to structured information. Based on the point cloud data of historical buildings, the component surface can be approximated by the Delaunay triangle mesh method, the surface model and topological relationship of historical building components are generated, as shown in Figure 4.

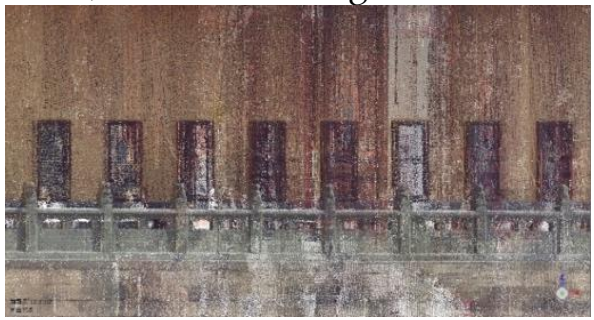

(a)

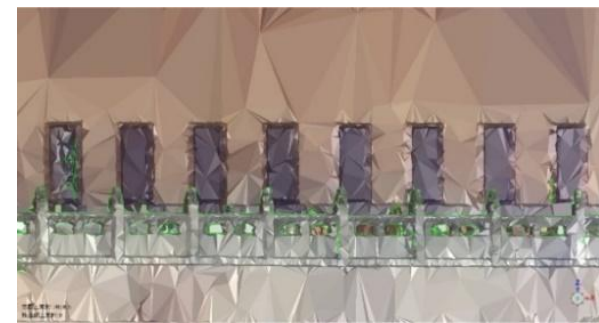

(b)

Figure 4: (a) A part of point cloud. (b) A part of polygon mesh generated.

\subsubsection{Reference Modeling}

Reference modeling is the process of reconstructing a geometric model based on polygon mesh data. 3DS Max is often employed to finish this step due to its powerful modeling and rendering function.

Components of a historical building can be divided into two categories by the geometry complexity, i.e., the core category contains the main building and structural components, such as walls, columns, roofs, etc., most of these components are the splicing or deformation of basic geometry, which can be created by 3DS Max's polygon modeling function, as shown in Figure 8; the affiliated category contains the decorative parts in historical buildings, such as mural, Buddha statues, etc. These components are completely irregular objects, and it is difficult to manually model directly. The only way is to import the polygon mesh into the 3DS Max software for fusion, and the polygon mesh is created by the method explained in previous part, as shown in Figure 6-7.

\subsubsection{Texture Mapping}

After the mesh model is transformed into a solid model, in order to improve the fidelity of the model, texture mapping is usually required. Texture mapping is the mapping of color on texture image to the corresponding part of solid model's surface depending on the relationship between the point cloud's intensity information and digital image's color information. In 3DS Max, the material editor and UVW map modifier can be used to complete the mapping, Figure 8 shows the overall model of the Mahavira Hall after the texture mapping, and the reconstruction of the geometric model is completed.
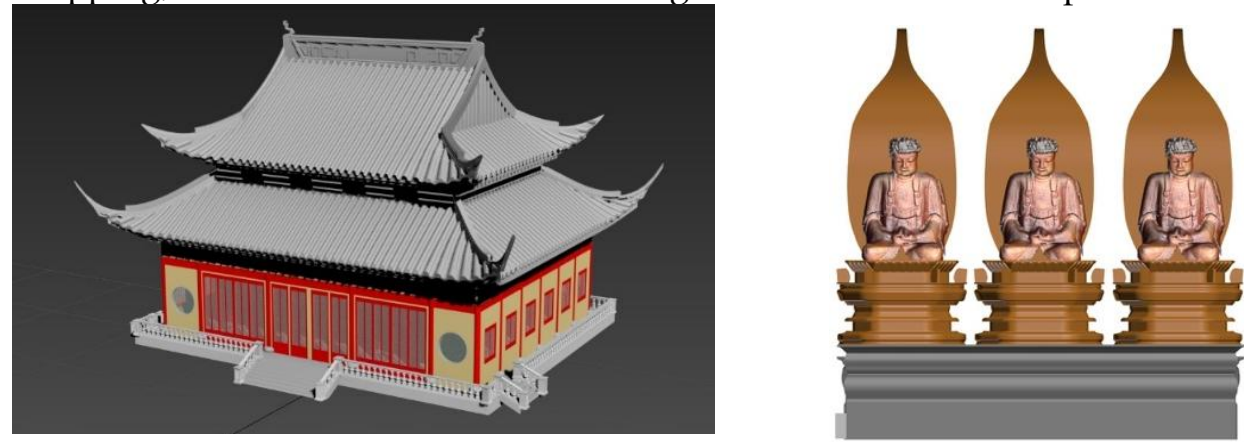

Figure 5: 3DS Max model of the Mahavira Hall. Figure 6: Three Buddhas' models 


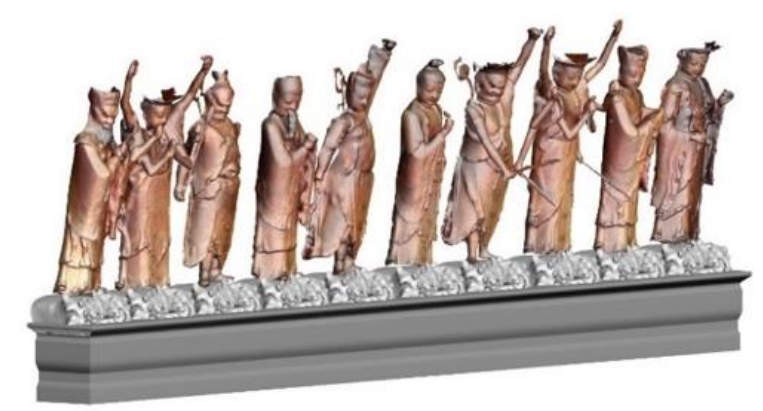

Figure 7: Twenty Heaven Statues' models.

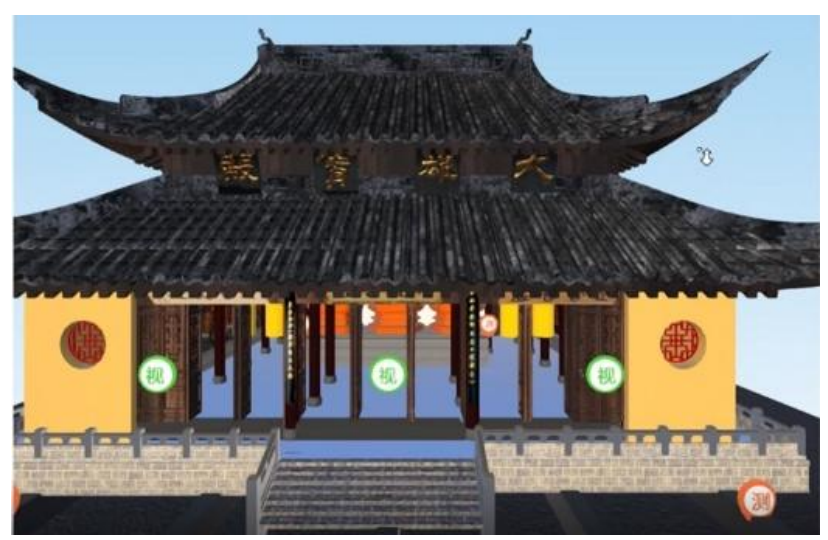

Figure 8: The textured model of the Mahavira Hall.

\section{PRINCIPLE OF AUTOMATIC MODEL TRANSFORMATION}

The geometric model of historical buildings contains a lot information of point, line, and area, most of which is not necessary for the description of the model, so data compression is needed. Moreover, it is difficult for the geometric model to incorporate other building information and its format is also difficult to meet sharing requirements.

In order to solve the above problems, an automatic conversion program is developed based on MAXScript and Revit API to convert the geometric model in 3DS Max into the information model in Revit. Revit was chosen here because it supports subsequent extensions based on the IFC standard.

The conversion program includes four steps: identification and classification of component types, geometric information extraction, element creation, and information attachment.

\subsection{Identification and Classification of Component Types}

\subsubsection{Main Structural Components-Geometric Recognition}

The main structural component types of historical buildings include beams, columns, walls, and slabs. Most of these components have their own standard geometry which size characteristics are easy to be identified. So, the type of the component can be determined by the geometry type and size. For example:

The wall in the 3DS Max model belongs to the cuboid class. Its width is generally less than $300 \mathrm{~mm}$ and its height is greater than $1000 \mathrm{~mm}$. After identification, the components could be stored in the corresponding array.

The geometry type of the rectangular beam belongs to the cuboid class. Its width is generally less than $500 \mathrm{~mm}$, and its height is less than $1000 \mathrm{~mm}$, and its length is more 
than three times of the height. Define an array named as "rectangularBeam", which can record these rectangular beams.

The geometry type of floor slab belongs to the cuboid class, while its width is generally greater than $1000 \mathrm{~mm}$, and its height is generally less than $200 \mathrm{~mm}$. Define an array named as "Floor", which can record these floor slabs.

The geometry types of circular columns and circular beams belong to the cylinder class, and their height is generally greater than 3 times of the radius. A component which axis direction is vertical can be stored in an array called "circularcolumn". And one which axis direction is horizontal can be stored in an array called "circularBeam".

\subsubsection{Complex Components-Name Recognition}

The historical building also contains other various complex parts, such as roofs, murals, bucket arches, etc. Such parts have complex shapes, different model attributes and characteristics, which cannot be identified by geometry types and sizes. It is the only way to identify them by name.

The following MAXScript code traverses and identifies the objects whose name contains "Roof", "Mural", "BucketArch", or "StonePier", and then groups them into the corresponding array respectively, to realize the identification of components with complex shapes:

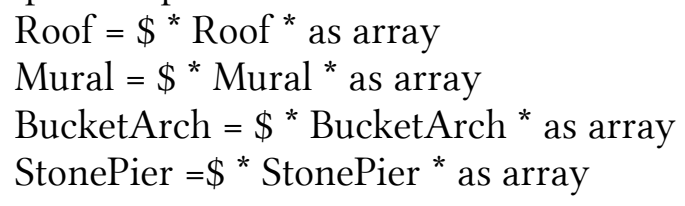

\subsection{Geometric Information Extraction - Clustering Algorithm}

For components with the same designed size, they definitely have small differences in the real size due to possible construction errors, measurement errors, or modeling errors. This not only brings trouble to the modeling, but also doesn't meet the requirements of application, so the size needs to be standardized by clustering algorithm.

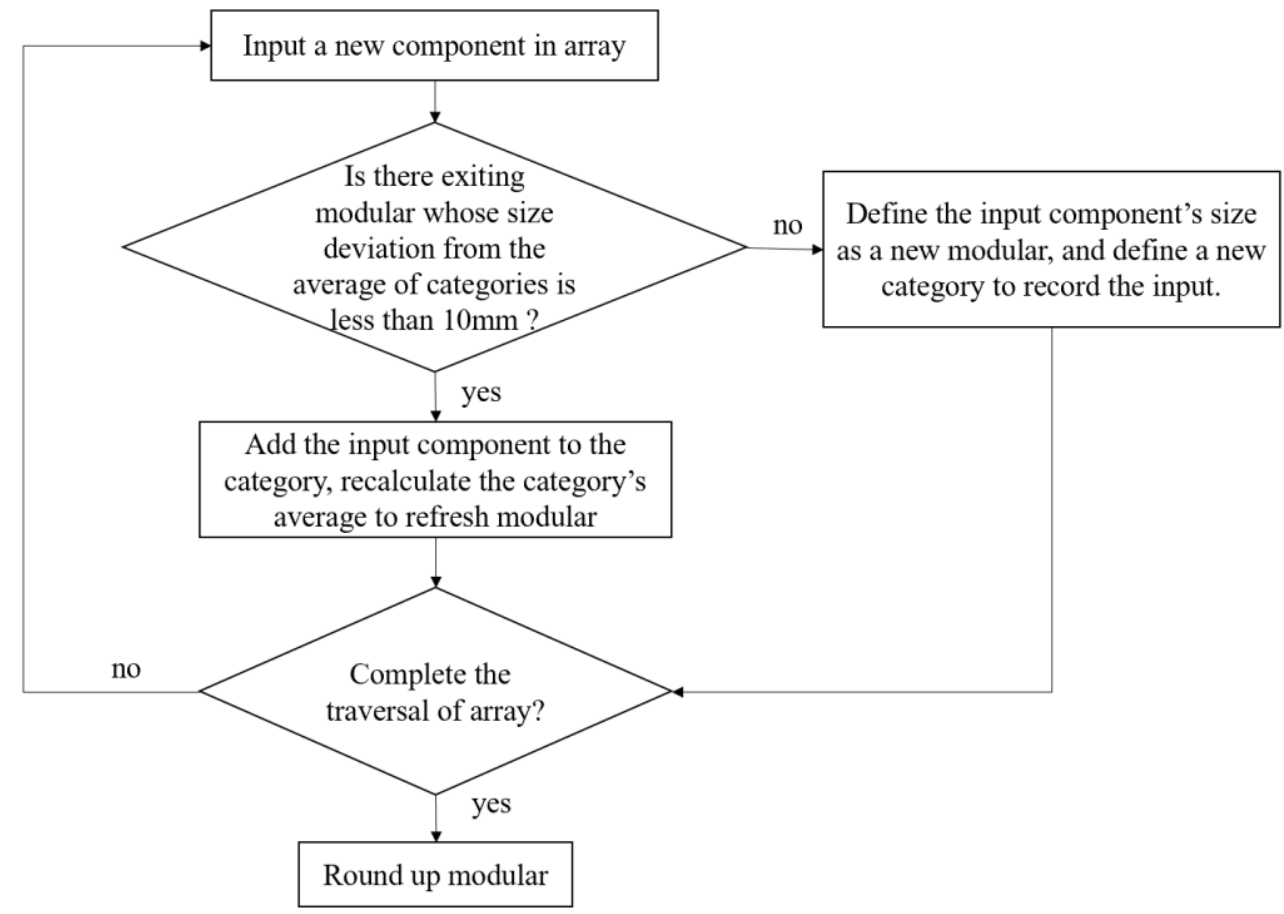

Figure 9: Flow chart of component clustering algorithm. 
In GB50206-2012, "Code for acceptance of construction quality of timber structures", the acceptable error for the manufacture and installation of beams, columns and other components are specified as $5 \mathrm{~mm}-10 \mathrm{~mm}$. Considering the crude of ancient construction technology, allowable error of geometric model can be appropriately enlarged to $10 \mathrm{~mm}$. That means size with errors not exceeding $10 \mathrm{~mm}$ can be standardized as a particular size which also can be called modular.

Therefore, for a specific component type, by traversing the component' array and clustering, components with the size of a deviation of less than $10 \mathrm{~mm}$ can be classified into one category, the flowchart is shown in Figure 9.

The obtained modular should be attached to the corresponding category and stored in a text file for modeling, while the original discrete data should be stored as the measured parameter values for subsequent information attachment and structural maintenance.

\subsection{Element Creation}

In Revit, the components such as columns, foundations, windows, and doors, can be created by calling the Revit API. So, by introducing the data information in the text file created before, a C \# program can fulfill element creation automatically.

The key problem is to obtain the parameter value imported into the creation function. After getting the necessary information, running the function can fulfill the whole creation, and the created elements need to be labeled for subsequent use.

\subsubsection{Creation of Reference Elements}

There are functions which can create reference elements, what is needed is elevation and grid information which can be identified and obtained from 3DS Max model.

Elevation value can be determined by the centroids' $\mathrm{Z}$ coordinate value of main structural member.

Grid refers to the planar positioning of the components, and the positioning axis is generally the centerline of the columns in a building plan. Therefore, the grid lines' information can be obtained by connecting the center point of the column. In order to facilitate the operation, the same arrangement of grid lines is arranged on each level.

\subsubsection{Instance Creation Based on Model Lines}

Components such as walls, slabs, and beams are created based on model lines. The required reconstruction information includes model lines, types, elevations, and structural features. Wall reconstruction is taken as a typical example, and the wall creation function is shown in Figure 10(a).

Analyze the parameters of the function one by one: "document" is the current Revit model file; "curve" represents the wall model line and determines the length of the wall, can be determined by the coordinates of the midpoints of the two short sides of the wall bottom; "wallTypeId" represents the wall type, determined by the wall width; "LevelId" represents the ID of the elevation where the wall is located, determined by reading the array that stores the elevation ID number when the elevation is created; "height" represents the height of the wall, determined by the height of the cuboid; "offset" represents the bottom offset, generally defaults to 0 ; "flip" represents whether to flip, the default is generally false; "structural" represents the wall structure, "true" represents the load-bearing wall, "false" represents the non-load-bearing wall, considering wood structures are generally filled walls, so the default is false.

Therefore, for each cuboid identified as a wall in 3DS Max, it is necessary to extract the coordinates of the two short sides of the bottom of the cuboid, the width of the 
cuboid, the height value, and the elevation number corresponding to the $\mathrm{Z}$ coordinate of the cuboid position. "wallTypeId" is determined by traversing and comparing width of each wall type with the width of the extracted cuboid, or in another word, determined by wall thickness.

\subsubsection{Instance Creation Based on Position Coordinates}

"NewFamilyInstance ()" in the Revit API provides methods for creating instances based on position coordinates of components. Take the creation of a cylinder column in Chinese traditional buildings as an example, its creation function in the Revit API is shown in Figure 10(b).

Analyze the information required one by one. For each cylinder identified as a column in 3DS Max, the program needs to extract the cylinder position coordinates, the cylinder radius and height, and the elevation corresponding to the cylinder position $\mathrm{Z}$ coordinate. "structuralType" is defined as "StructuralType.Column" by default. "symbol" is actually the column type, can be determined by traversing all the family types whose name string contains a "circular column" and comparing the radius and height of the cylinder.

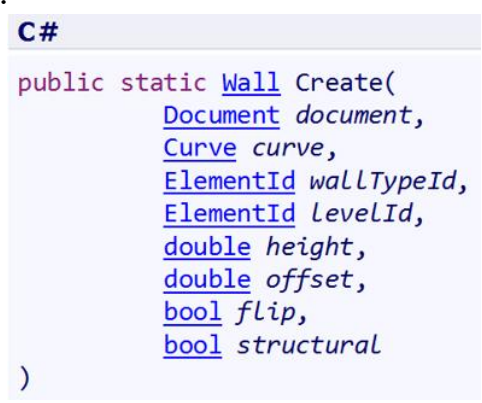

(a)

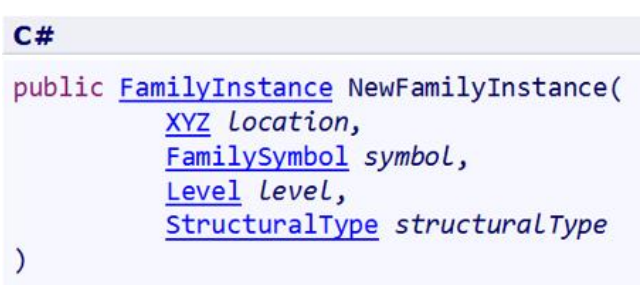

(b)

Figure 10: (a)Wall creation function (b)Column creation function.

\subsubsection{Instance Creation Based on Host}

The Revit API provides a host-based instance creating method, which is suitable for the creation of doors and windows embedded in the wall. Take the window as an example, its creation function is shown in Figure 11.

Analyze the information contained in the function one by one. For each object defined as a window in 3DS Max, you need to extract its position coordinates, the length, width, and height of the window, the corresponding number of the host wall to which the window belongs to, and the host wall. "structuralType" is defined as "StructuralType.NonStructural" by default. The length, width, and height of the window can be determined by traversing the coordinates of all the vertices of the window grid object to determine the XYZ direction range. "symbol" is actually the form of window, can be determined by traversing all the family types whose name string contains a "window" and comparing the length, width, and height of the form. The host wall and the corresponding elevation of the host wall may be determined by the ID numbers stored in the wall creation. 


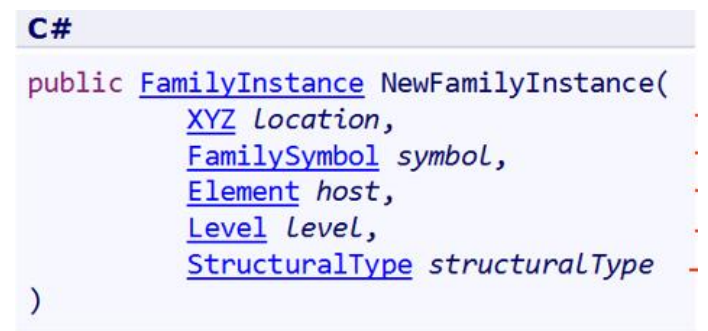

Figure 11: Window creation function.

\subsubsection{Supplementary Creation in GUI Interface}

The above methods have been able to meet most of the modeling requirements of historical buildings, but the Revit API does not provide all the data creation interfaces in Revit. Therefore, some components need to be created in GUI interface. For example, Buddha statues have complex and different shapes, and it is difficult to restore them perfectly using the family tools. However, such elements have little effect on the structural maintenance in the whole life cycle, and it is acceptable to import the relevant 3DS Max model into Revit as an external symbol and to fulfill the building information model manually.

\subsection{Automatic Information Expansion}

Nowadays, a large amount of historical building information, especially the structural inspection results, are stored in the form of electronic documents. It's not hard to extract information and attach them to BIM model by a computer program.

Shared parameters are parameter definitions that can be used in multiple families or projects, which are useful for information attachment. The procedure of the shared parameter definition, binding and parameter value setting is shown in Figure 12.

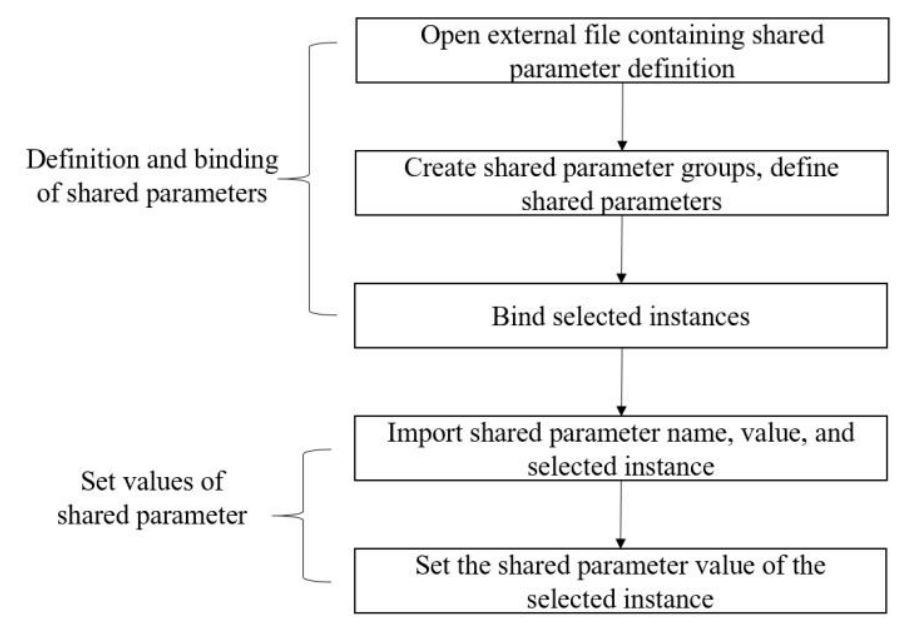

Figure 12: Shared parameter definition, binding and parameter value setting.

Based on the above principle, a C\# program is coded to realize the automatic information expansion. The main steps of the process are shown in Figure 13. 


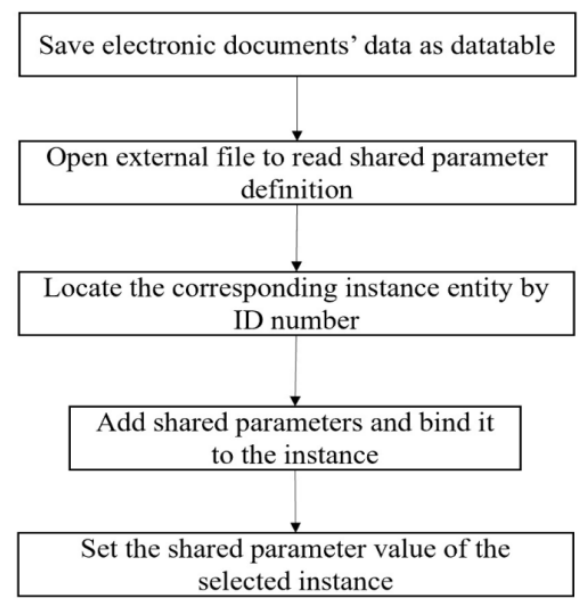

Figure 13: Flow chart of shared parameter definition and assignment.

\section{Application of Shanghai Jade Buddha Temple's INFORMATION MODEL}

Floor plans can be automatically generated from the Revit model, as shown in Figure 14; Panoramic map of the Jade Buddha Temple can be created using Pano2VR for tourism promotion and navigation, as shown in Figure 15.
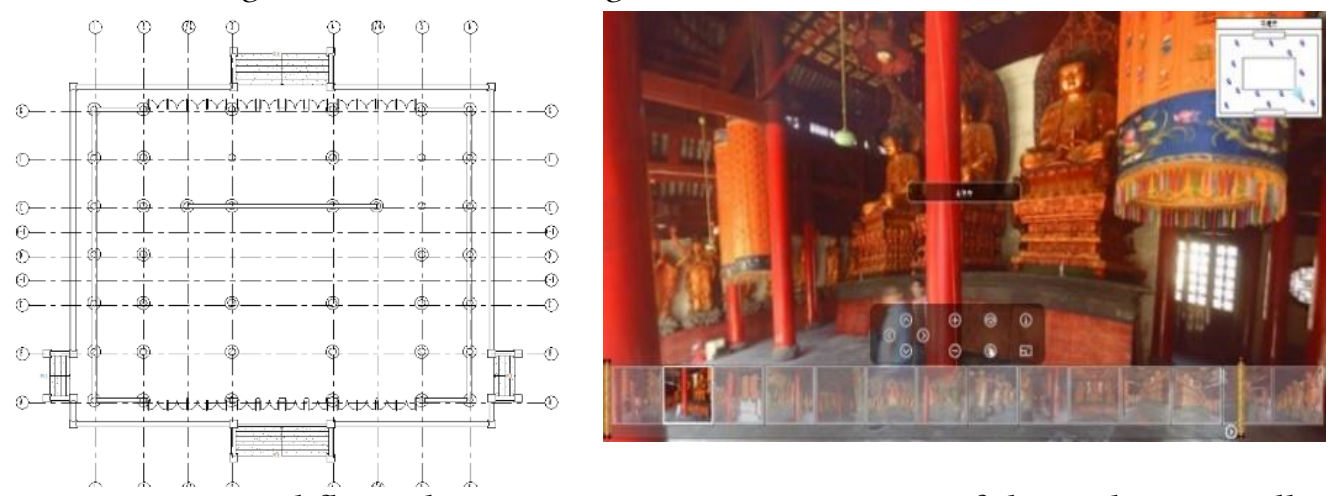

Figure 14: Ground floor plan. Figure 15: Panoramic map of the Mahavira Hall.

If the time-dependent information is integrated to the Revit model, so-called "4D" information model can be generated, which is useful for rehabilitation and maintenance throughout the life cycle. According to the historical documents of the Jade Buddha Temple, the Temple went through 7 repairs, 1 large-scale expansion and recent Panning. So, divided the information model of the Jade Buddha Temple into 12 stages on the time scale and the rehabilitation information can be integrated at the corresponding stage, as shown in Figure 16. 


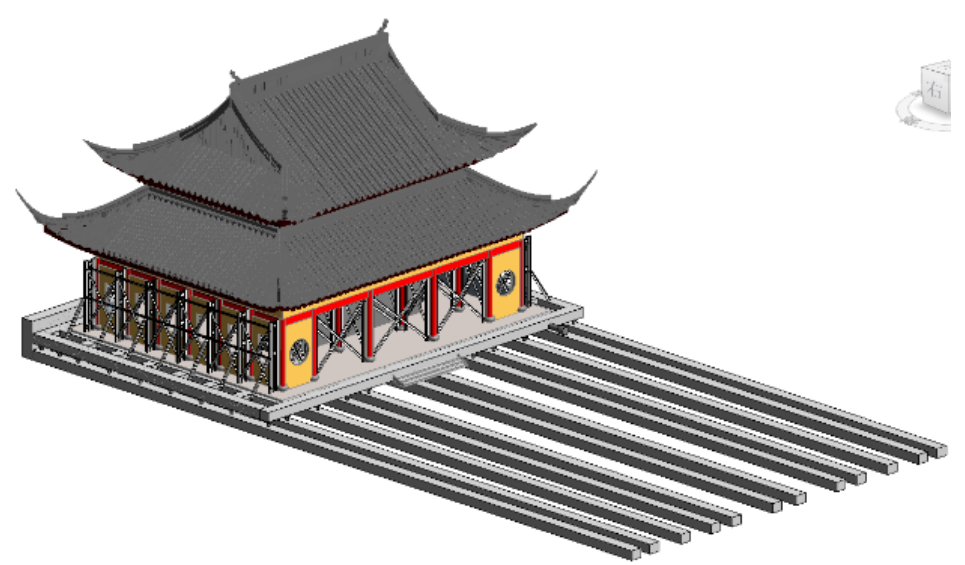

Figure 16: Model of the Mahavira Hall in Panning Stage.

\section{CONCLUSIONS}

In this research, multi-source data collecting, processing and fusion for 3D reverse modeling were performed, and geometric model was created by reference modeling. Then, its BIM model was generated by the automatic conversion program which can convert the geometric model in 3DS Max into the information model in Revit and attach information, because BIM model can not only reduce data size, but also increase the amount of model information and improve the generality of the model. The application on Shanghai Jade Buddha Temple successfully verify the feasibility of the procedure and demonstrates the high potential of the workflow on lifecycle management of historical building.

However, problem still remains: reference modeling rely on a lot of manual operations. To solve this problem, future research will focus on semantic segmentation of point clouds using deep learning, which can separate all components from each other in point cloud model. This is helpful for component identification and geometric information extraction directly from segmented point cloud, which make automatic conversion from point cloud into the information model possible.

\section{REFERENCES}

Agarwal, S., Furukawa, Y., Snavely, N., Simon, I., Curless, B., Seitz, S.M., and Szeliski, R. (2011). Building Rome in a Day. Commun. ACM, 54(10), pp. 105-112, https://doi.org/10.1145/2001269.2001293, 105-112.

Bhatla, A., Choe, S.Y., Fierro, O., and Leite, F. (2012). Evaluation of accuracy of as-built 3D modeling from photos taken by handheld digital cameras. Autom. Constr., 28, pp. 116-127, https://doi.org/10.1016/j.autcon.2012.06.003, 116-127.

Biagini, C., Capone, P., Donato, V., and Facchini, N. (2016). Towards the BIM implementation for historical building restoration sites. Autom. Constr., 71, pp. 7486, https://doi.org/10.1016/j.autcon.2016.03.003, 74-86.

Crespi, P., Franchi, A., Ronca, P., Giordano, N., Scamardo, M., Gusmeroli, G., and Schiantarelli, G. (2015). From BIM to FEM: the analysis of an historical masonry building, in: Building Information Modelling (BIM) in Design, Construction and Operations, 1, pp. 581-592, https://doi.org/10.2495/bim150471, 581-592.

Eslami, A., Ronagh, H.R., Mahini, S.S., and Morshed, R. (2012). Experimental investigation and nonlinear FE analysis of historical masonry buildings - A case 
study. Constr. Build. Mater., 35, pp. 251-260, https://doi.org/10.1016/j.conbuildmat.2012.04.002, 251-260.

Golparvar-Fard, M., Bohn, J., Teizer, J., Savarese, S., and Peña-Mora, F. (2011). Evaluation of image-based modeling and laser scanning accuracy for emerging automated performance monitoring techniques. Autom. Constr., 20, pp. 1143-1155, https://doi.org/10.1016/j.autcon.2011.04.016, 1143-1155.

Klein, L., Li, N., and Becerik-Gerber, B. (2012). Imaged-based verification of as-built documentation of operational buildings. Autom. Constr., 21, pp. 161-171, https://doi.org/10.1016/j.autcon.2011.05.023, 161-171.

Lee, K. H., Woo, H. and Suk, T. (2001). Data reduction methods for reverse engineering. International Journal of Advanced Manufacturing Technology, 17(10), pp. 735-743, https://doi.org/10.1007/s001700170119, 735-743.

Lerma, J.L., Navarro, S., Cabrelles, M., and Villaverde, V. (2010). Terrestrial laser scanning and close range photogrammetry for 3D archaeological documentation: the Upper Palaeolithic Cave of Parpalló as a case study. J. Archaeol. Sci., 37(3), pp. 499507, https://doi.org/10.1016/j.jas.2009.10.011, 499-507.

López, F.J., Lerones, P.M., Llamas, J., Gómez-García-Bermejo, J., and Zalama, E. (2018). A review of heritage building information modeling (H-BIM). Multimodal Technol. Interact., 2(2), https://doi.org/10.3390/mti2020021.

Saygi, G., Agugiaro, G., Hamamcloğlu-Turan, M., and Remondino, F. (2013). Evaluation of GIS and BIM roles for the information management of historical buildings. ISPRS Annals of the Photogrammetry, Remote Sensing and Spatial Information Sciences. Copernicus GmbH, 2(5), pp. 283-288, https://doi.org/10.5194/isprsannals-II-5-W1283-2013, 283-288.

Theiler, M., and Smarsly, K. (2018). IFC Monitor-An IFC schema extension for modeling structural health monitoring systems. Advanced Engineering Informatics, 37, pp. 5465, http://doi.org/10.1016/j.aei.2018.04.011, 54-65.

Torres-Martínez, J.A., Seddaiu, M., Rodríguez-Gonzálvez, P., Hernández-López, and D., González-Aguilera, D. (2015). A Multi-data source and multi-sensor approach for the $3 \mathrm{~d}$ reconstruction and visualization of a complex archaelogical site: The case study of tolmo de minateda. International Archives of the Photogrammetry, Remote Sensing and Spatial Information Sciences - ISPRS Archives. International Society for Photogrammetry and Remote Sensing, 40(5W4) pp. 37-44, https://doi.org/10.5194/isprsarchives-XL-5-W4-37-2015, 37-44.

Wang, S.H., Wang, W.C., Wang, K.C., and Shih, S.Y. (2015). Applying building information modeling to support fire safety management. Autom. Constr., 59, pp. 158-167, https://doi.org/10.1016/j.autcon.2015.02.001, 158-167.

Xu, Z., Wu, L., Shen, Y., Li, F., Wang, Q., and Wang, R. (2014). Tridimensional reconstruction applied to cultural heritage with the use of camera-equipped UAV and terrestrial laser scanner. Remote Sens., 6(11), pp. 10413-10434, https://doi.org/10.3390/rs61110413, 10413-10434.

Yastikli, N. (2007). Documentation of cultural heritage using digital photogrammetry and laser scanning. J. Cult. Herit., 8(4), pp. 423-427. https://doi.org/10.1016/j.culher.2007.06.003, 423-427. 